\title{
The Review of Intelligent Mechanical Engineering Based on Artificial Neural Network
}

\author{
Yue LIU ${ }^{1, a}$ \\ ${ }^{1}$ Tianjin Transportation Vocational College, Tianjin 300110,China \\ ayueliu@163.com
}

Keywords: Artificial Neural Network, BP neural network, Hopfield neural network, SOM network, Intelligent Mechanical Engineering

\begin{abstract}
The application of artificial neural network theory has penetrated into all fields, and in the intelligent control, the development of the neural network computer optimization computing, computer vision and image processing, pattern recognition, continuous speech recognition, data compression, knowledge processing showed its great application value, has made encouraging progress. In the field of mechanical fault diagnosis, the application of artificial neural network is still in the stage of development. In this paper, the intelligent diagnosis of machine faults, the mechanical structure analysis and geometry modeling and mechanical design, optimization of the application of artificial neural network technology in some studies. . With the development of the theory and perfect, will be on the development of intelligent mechanical engineering plays a more important role.
\end{abstract}

\section{Introduction}

Artificial Neural Network is a very rapid development of cross subject, it comes to biology, computer science, mathematics, electronics, physics, psychology, cognitive science and many other subjects. It is the study of the adaptive programming information system of an engineering discipline, is actually a new information processing technology. Its principle is on the basis of modern neuroscience research results, using huge amount of parallel, segmental storage and simulation approach, trying to reflect some of the basic characteristics of the human brain information processing [1-2]. With the development of the theory and perfect, will of the development of intelligent mechanical engineering plays a more important role.

With the application of modern science and technology on the device, the structure of the modern mechanical equipment more and more complex, function more and more complete, more and more is also high degree of automation. Artificial neural network has extensive application in the field of mechanical engineering [3]. From the basis of the mechanical engineering disciplines to the machine design, manufacture and operation, artificial neural network technology is pervasive, so to speak. In this paper, the intelligent diagnosis of machine faults, the mechanical structure analysis and geometry modeling and mechanical design, optimization of artificial neural network technology in the application of some review. As you might expect, with the passage of time and research thorough, the artificial neural network technology in mechanical engineering will also appear more applications in the field of research subject.

\section{Artificial neural network model applied in the field of mechanical engineering}

BP neural network. BP neural network is multilayer feed forward neural network based on BP algorithm, it is D.E. Rumelhart, J.L. McCellnad and their team studied and designed in 1986 [4]. Currently, the practical application of artificial neural networks, the vast majority of the neural network model is BP and its variants, it is also a former core network, reflecting the most essential part of the artificial neural network. BP neural network is a multi-layer network, the input layer and output layer, hidden layer (layer or multi-layer structure, a typical three-layer BP neural network 
model shown in Figure 1. The conversion function is a hidden layer nonlinear function. Nonlinear transformation function of the output layer, also may be linear, the input, output mapping needs.

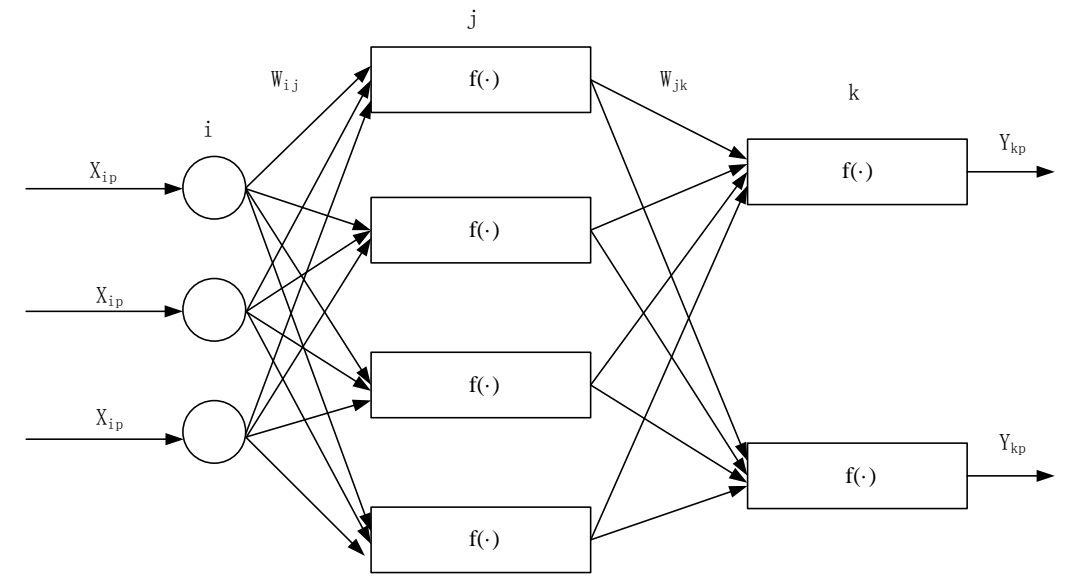

Figure 1. Typical BP neural network model

The main idea of back-propagation learning algorithm is divided into two stages: the first stage (active dissemination), enter the information step by step, the state of each layer of neurons compute the actual output value of each unit from the input layer affects only the beginning next layer neuron state; a second phase (back propagation) process, if the output layer fails to achieve the desired output value, actual output stratified recursively calculates the difference between the desired output value, according to the previous error correction layer the weight of the error signal to a minimum. By direction of the error function is continuous with respect to changes in the network weights and bias slope down, closer to our goal. Each weight value and the error variance is proportional to the impact of network errors.

Hopfield neural network. Hopfield neural network is a neural network model .Hopfield recurrent neural network, there is a feedback from the output to the input connection. Hopfield network has discrete and continuous two. Feedback neural network feedback because of its output to its input; therefore, Hopfield network Inspired input will produce constant state of change [5]. When input you can then remove the output Hopfield's request, the output back to the input to produce a new output, the feedback process has to proceed. If Hopfield network is a stable network can converge, then the change feedback and iterative calculation process produced less and less, once it reaches a stable equilibrium state; then Hopfield network will output a steady constant. For a Hopfield network, the key is to make sure it is right in a stable condition coefficient. Hopfield neural network structure shown in Figure 2.

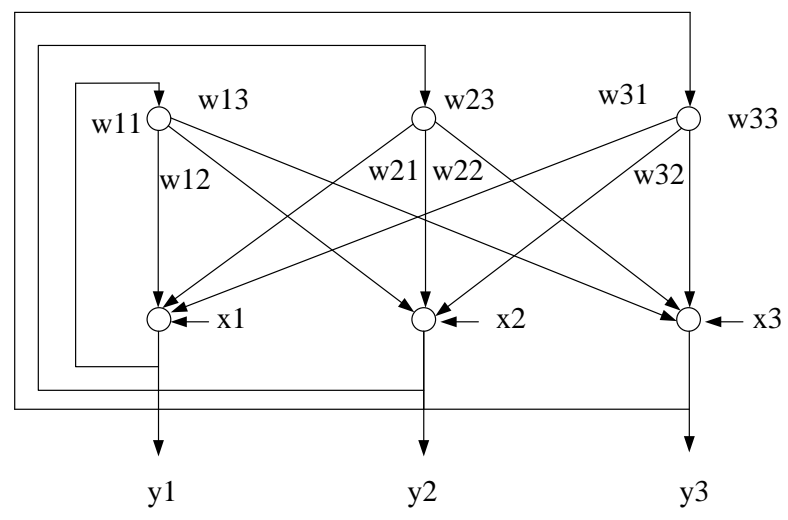

Figure 2. Hopfield neural network structure

SOM network. SOM network put forward by Kohonen when Finland scholars in 1981 is a kind of self-organizing competitive neural network, the input layer and output layer (competition), typical structure as shown in figure 3. Neural input layer number is $\mathrm{m}$, the competitive layer composed of neurons two-dimensional planar array, the input layer and competition to all 
connections between the neurons. Before the start of the training, the output contacts are endowed with a small random weighting. After the input samples, make the output contact to compete, win and adjust the contact and its neighborhood within the contact of the weights. Training has been completed, the output layer nodes distribution can keep data in meta-space topological distribution [6-7].

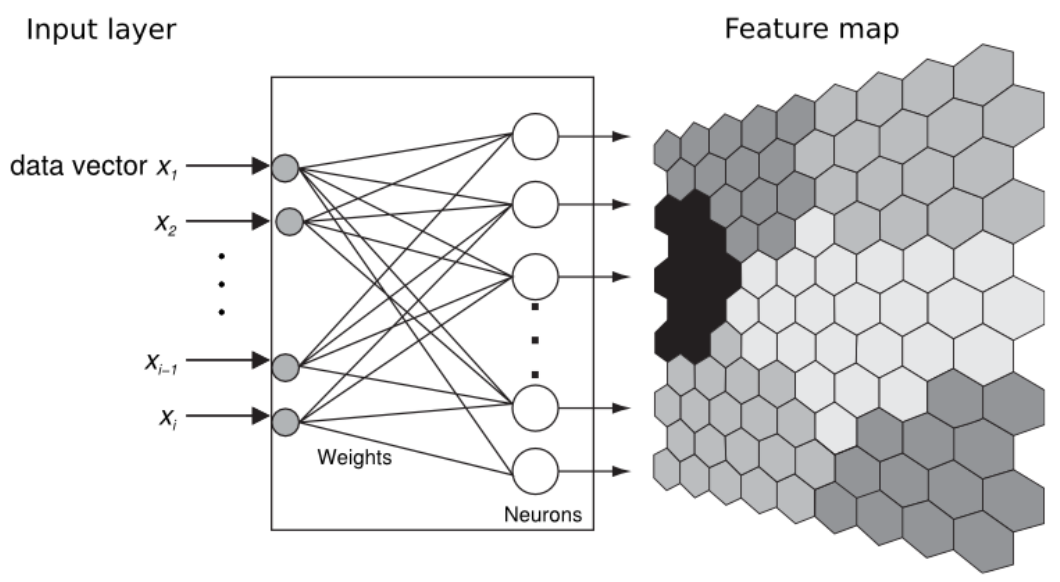

Figure 3.SOFM network structure

SOM network training process using self-organizing competitive learning principles, its training process is as follows:

(1) Network initialization. M randomly input neurons to the output neuron connection weights given smaller weights. Defines the maximum train length (typically define an appropriate convergence criteria more difficult, so much by defining a maximum length of training as a condition for the end);

(2) Determine the best matching unit. Most from the training data set to select a sample $\mathrm{x}$, compute the Euclidean distance between the sample and each output node (also use other distance), select the output sample $\mathrm{x}$ nearest node, the node is called the input samples good matching unit, and gives its adjacent neurons collections:

(3) Weight values learning. Right field function according to pre-defined node to determine the best matching unit in the neighborhood, and the neighborhood node BMU adjustment values:

(4) Whether reached a preset requirements. If the training has not reached the maximum length, the process returns to step (2) to continue the training, otherwise the end of the training

\section{Application of artificial neural network in mechanical fault diagnosis}

Mechanical Fault Diagnosis is the use of acquisition in the operation of the machinery and equipment in or under the condition of relatively static state information, the analysis of the measured signal and processing, and combining with the history of the diagnostic object state, to quantitatively identify technology of real-time state of Mechanical equipment and parts, and predict the abnormal failure and predict the future state of technology, technology to determine the necessary countermeasures [8]. Its basic principle is to use the new achievement of all the contemporary science and technology, discovered the hidden trouble of mechanical equipment, in order to equipment accident prevention. Fault diagnosis of mechanical equipment, in fact, ever since the dawn of industrial production, the touch of early people according to the equipment, to sound and vibration state characteristics of feelings, with the experience of the craftsman, can determine the existence of some faults, and repair measures are put forward. Mechanical fault diagnosis technology is the product of the development of modern production, is developed on the basis of equipment management and maintenance.

Fault diagnosis is the key to realize the fault symptom space and fault space mapping, so as to realize the fault identification and diagnosis. The traditional method is based on symbolic reasoning expert system. But the expert system for fault diagnosis knowledge acquisition difficulties matching 
conflict, combinatorial explosion and insurmountable problems, such as, make its application cannot reach the desired effect. Self-learning ability of artificial neural network, non-linear mapping ability, parallel computing ability and fault tolerance for constructing a new fault diagnosis system provides powerful means. Based on artificial neural network fault diagnosis system, is to use as input, the credibility of fault symptoms after artificial neural network of parallel numerical calculation, the output corresponding to the credibility of the fault. Its composition generally includes man-machine interface module, knowledge base and diagnosis reasoning, diagnostic information acquisition module, system learning module and consulting module.

\section{Artificial neural network application in the mechanical structure analysis and geometric modeling}

Structure analysis can realize the design parameters to modal parameter mapping relationship, the traditional method is finite element analysis, now also someone with artificial neural network to realize the mapping. In some of the structure of the mature experience of data or have been obtained by the finite element analysis results to train the BP network, the cumulative value of the training and then use the network structure analysis. The structure dynamic design is to realize the modal parameters to the mapping relationship of design parameters, this is the inverse process of structure analysis, the solution difficult. Some scholars try to use artificial neural network to implement this function. One of the specific approach is to use finite element structure analysis of the data to train the BP network, which USES modal parameters as the input of artificial neural network, and design parameters as the output, so as to realize the mapping relationship between them, and then used for structural dynamic design and modification.

Geometric modeling, especially the complex surface modeling, generally adopt the method of spline function fitting. This method is complicated boundary conditions, modify the disadvantages such as difficult, large amount of calculation. The nonlinear mapping ability of artificial neural network used in geometric modeling is a new attempt. With known type of particle surface of X, Y coordinates as input, $\mathrm{Z}$ as the output, choose appropriate training of artificial neural network training sample set, which can realize the arbitrary curved surface modeling. This method is especially suitable for modeling based on measurement.

\section{Artificial neural network application in mechanical design and optimization}

Modern mechanical system structure is complicated, for its static properties (strength, stiffness, etc.) and the dynamic performance (displacement, velocity, acceleration response, the structure of the system natural frequency and vibration mode) of integrated optimization problem, in terms of scale and mathematical calculation complexity are all unprecedented. For this kind of size optimization, topology optimization and performance optimization of global optimization problem, traditional optimization theory based on mathematical programming method is difficult to make a difference.

Due to the minimum energy of the Hopfield net always tends to a stable state, this kind of global optimization problem can be converted into artificial neural network energy function solution of the minimum point. The artificial neural network dynamic system as the stability of the attractor corresponding generalized objective function is minimum point, from the initial point (that is, the power system corresponding to the artificial neural network of initial conditions), the artificial neural network to hope that the minimum point at the same time, complete the optimization calculation. Application is the key to apply the actual problem in your field of expertise into optimization problem, and then construct a suitable network structure and parameters, and the right energy function to realize optimization.

\section{Conclusion}

In the field of mechanical engineering, there have been many researchers on the basis of neural network theory, method, technique and its application has done a lot of pioneering or exploratory 
work, a lot of achievements. But, look from domestic actual situation, theory, method and technology of artificial neural network in the application and development in the field of mechanical engineering, is still in the preliminary stage. At present, there are two opposite views: one is that the artificial neural network has been research for many years, no more advanced and novel, this is a kind of stagnation and inaction; The other opposite, always think of artificial neural network is a theoretical research work, not for actual production. For these two views, in particular the right decisions at this stage, comprehensive planning and positive guidance, upgrading of machinery products, machinery industry for the future to contribute to the revitalization and take-off of, also can make the artificial neural network in the field of mechanical engineering with new opportunities and greater development.

\section{References}

[1] Sick B. On-line and indirect tool wear monitoring in turning with artificial neural networks: a review of more than a decade of research[J]. Mechanical Systems and Signal Processing, 2002, 16(4): 487-546.

[2] Choudhary A K, Harding J A, Tiwari M K. Data mining in manufacturing: a review based on the kind of knowledge[J]. Journal of Intelligent Manufacturing, 2009, 20(5): 501-521.

[3] Chandrasekaran M, Muralidhar M, Krishna C M, et al. Application of soft computing techniques in machining performance prediction and optimization: a literature review $[\mathrm{J}]$. The International Journal of Advanced Manufacturing Technology, 2010, 46(5-8): 445-464.

[4] Quintana G, Garcia-Romeu M L, Ciurana J. Surface roughness monitoring application based on artificial neural networks for ball-end milling operations[J]. Journal of Intelligent Manufacturing, 2011, 22(4): 607-617.

[5] Karaboga D, Akay B. A survey: algorithms simulating bee swarm intelligence[J]. Artificial Intelligence Review, 2009, 31(1-4): 61-85.

[6] Dounis A I, Caraiscos C. Advanced control systems engineering for energy and comfort management in a building environment-A review $[\mathrm{J}]$. Renewable and Sustainable Energy Reviews, 2009, 13(6): 1246-1261.

[7] Chen C W, Chen P C, Chiang W L. RETRACTED: Modified intelligent genetic algorithm-based adaptive neural network control for uncertain structural systems[J]. Journal of Vibration and Control, 2013, 19(9): 1333-1347.

[8] Mohanraj M, Jayaraj S, Muraleedharan C. Applications of artificial neural networks for refrigeration, air-conditioning and heat pump systems-A review[J]. Renewable and Sustainable Energy Reviews, 2012, 16(2): 1340-1358. 